\title{
VIEWPOINT
}

\section{YAP-Hippo signalling downstream of leukemia inhibitory factor receptor: implications for breast cancer}

\author{
Alexander Hergovich*
}

\begin{abstract}
The proto-oncogenes YAP and TAZ have previously gained much attention as downstream effectors of Hippo tumour suppressor signalling. While the regulation of YAP/TAZ by MST/LATS kinases is reasonably well understood, the nature of factors functioning upstream of MST/LATS is yet to be elucidated in detail. A recent paper by Ma and co-workers defines a novel role for leukemia inhibitory factor receptor (LIFR) signalling upstream of the Hippo-YAP pathway in breast cancer metastasis. Moreover, a whole genome in vivo RNA interference screen by Lippmann and colleagues identified LIFR as a breast tumour suppressor. Here, we discuss the implications of these studies for breast cancer research and treatment.
\end{abstract}

\section{Background}

Hippo signalling is a tumour suppressor cascade highly conserved from yeast to man [1]. In mammals, Hippo signalling is deregulated in various cancers; hence, mammalian Hippo signalling has gained much attention over the past years [2]. In a nutshell, the canonical Hippo pathway functions as follows: activated MST1/2 kinases (mammalian Ste20-like serine/threonine kinase 1/2) phosphorylate hMOB1 (human Mps one binder 1) and LATS1/2 (large tumour suppressor serine/threonine kinase $1 / 2$ ), resulting in the formation of an active hMOB1-LATS complex that phosphorylates the protooncogenes YAP (Yes-associated protein) and TAZ (transcriptional co-activator with PDZ-binding motif), which finally leads to the accumulation of inactive cytoplasmic YAP/TAZ [3].

YAP is overexpressed in various human cancers [4,5], supporting a role for it as a proto-oncogene. In breast

${ }^{*}$ Correspondence: a.hergovich@ucl.ac.uk

UCL Cancer Institute, University College London, WC1E 6BT, London, UK cancer, however, gain or loss of YAP expression has been reported [6-9], suggesting that YAP might have oncogenic and tumour suppressive functions dependent on the breast cancer subtype. TAZ is overexpressed in breast cancer $[10,11]$, but a recent report [12] suggests also a tumour suppressive role for TAZ. Therefore, the roles of YAP/TAZ-Hippo signalling in breast cancer are debatable. Considering that metastases at distant sites, and not the primary breast tumour, are the main cause of death, we must further consider YAP/TAZ functions in metastasis, as highlighted by a recent report by $\mathrm{Ma}$ and colleagues [13].

\section{The articles}

To uncover novel factors involved in the initiation/ progression of tumours, Lippman and colleagues [12] screened in vivo the entire human genome by RNA interference, thereby identifying LIFR as a novel tumour suppressor. Silencing of LIFR was sufficient to transform normal mammary cells, and reciprocally, LIFR overexpression in breast cancer cells suppressed tumour growth [12], suggesting that LIFR is a clinically important breast tumour suppressor. However, Iorns and colleagues [12] did not define how LIFR functions as a tumour suppressor.

In parallel, $\mathrm{Ma}$ and co-workers discovered a role for LIFR as a novel breast cancer metastasis suppressor [13]. In full agreement with Iorns and colleagues [12], they also found that LIFR is downregulated in breast cancer [13], but controversially reported that LIFR silencing did not affect primary tumour growth [13]. However, overexpression of LIFR in metastatic breast cancer cell lines dramatically reduced metastases formation [13]. Furthermore, Ma and colleagues investigated the mechanisms downstream of LIFR. Based on a recent report [14] linking LIF (the ligand for LIFR) to the regulation of YAP, they examined the role of LIFR in YAP-Hippo signalling. Unlike in embryonic stem cells [14], addition of LIF resulted in increased YAP phosphorylation in breast cancer cell lines, thereby resulting in the inactivation of YAP [13]. Since phosphorylation of MST/LATS was 
increased upon LIFR overexpression [13], it is possible that the effect on YAP is driven by canonical Hippo signalling. Moreover, they provided evidence suggesting that LIFR signals to MST/LATS via Scribble [13], an adaptor that can link MST/LATS/YAP/TAZ complexes [11].

\section{The viewpoint}

Two recent reports highlight LIFR as a novel player in breast cancer. The work by Iorns and colleagues [12] defines LIFR as a breast tumour suppressor, while Ma and co-workers [13] define LIFR as a breast cancer metastasis suppressor. Current evidence strongly suggests that LIFR functions by inhibiting YAP [13]. This novel role for YAP in breast cancer metastasis is supported by a recent paper from the Hynes laboratory [15], but the involvement of canonical Hippo signalling is not so evident. They show that LIFR overexpression correlates with increased LATS1 phosphorylation, while YAP(S112A) drives metastases despite LIFR overexpression [13]. This suggests that LIFR triggers YAP phosphorylation by activating LATS1. However, given that YAP phosphorylation appears to be independent of LATS $1 / 2$ in other cancer settings [16], it will be important to confirm the identity of the kinase(s) targeting YAP in these settings before we can make final conclusions.

Considering that TAZ-Hippo signalling is already implicated in breast cancer $[10,11]$, it is likely that LIFR also functions upstream of TAZ. In particular, it will be interesting to determine whether the recently reported role for TAZ in breast cancer metastasis [17] is controlled by LIFR. However, Iorns and colleagues identified TAZ (WWTR1) as a potential breast tumour suppressor in their screen [12]. At first glance, these observations do not seem to make sense, but as already speculated for YAP [4,6-9], TAZ might have oncogenic and tumour suppressive functions dependent on the breast cancer subtype or progression stage, a phenomenon already reported for other factors in different cancer types [18]. Since increased YAP/TAZ levels correlate with taxol resistance $[7,19]$, YAP/TAZ have been considered as targets/biomarkers in breast cancer. Based on the work by the Ma and Lippman laboratories [12,13], however, LIFR activation appears to be the more attractive clinical target for the treatment of breast cancer, since the roles of YAP/TAZ-Hippo signalling in breast cancer subtypes are yet to be defined in more detail.

\section{Abbreviations}

LIF, leukemia inhibitory factor; LIFR, leukemia inhibitory factor receptor.

\section{Acknowledgements}

We thank J Lisztwan and C Gewinner for their feedback on this manuscript. This work was supported by the Wellcome Trust grant 090090/Z/09/Z.

Published: 5 December 2012

\section{References}

1. Hergovich A, Stegert MR, Schmitz D, Hemmings BA: NDR kinases regulate essential cell processes from yeast to humans. Nat Rev Mol Cell Biol 2006, 7:253-264.

2. Pan D: The hippo signaling pathway in development and cancer. Dev Cell 2010, 19:491-505.

3. Zhao B, Tumaneng K, Guan KL: The Hippo pathway in organ size control, tissue regeneration and stem cell self-renewal. Nat Cell Biol 2011, 13:877-883.

4. Hergovich A, Hemmings BA: Mammalian NDR/LATS protein kinases in hippo tumor suppressor signaling. Biofactors 2009, 35:338-345.

5. Hong W, Guan KL: The YAP and TAZ transcription co-activators: Key downstream effectors of the mammalian Hippo pathway. Semin Cell Dev Biol 2012, 23:785-793.

6. Matallanas D, Romano D, Yee K, Meissl K, Kucerova L, Piazzolla D, Baccarini M, Vass JK, Kolch W, O'Neill E: RASSF1A elicits apoptosis through an MST2 pathway directing proapoptotic transcription by the p73 tumor suppressor protein. Mol Cell 2007, 27:962-975.

7. Overholtzer M, Zhang J, Smolen GA, Muir B, Li W, Sgroi DC, Deng CX, Brugge JS, Haber DA: Transforming properties of YAP, a candidate oncogene on the chromosome 11q22 amplicon. Proc Natl Acad Sci U S A 2006, 103:12405-12410.

8. Wang X, Su L, Ou Q: Yes-associated protein promotes tumour development in luminal epithelial derived breast cancer. Eur J Cancer 2012, 48:1227-1234.

9. Yuan M, Tomlinson V, Lara R, Holliday D, Chelala C, Harada T, Gangeswaran R, Manson-Bishop C, Smith P, Danovi SA, Pardo O, CrookT, Mein CA, Lemoine NR, Jones LJ, Basu S: Yes-associated protein (YAP) functions as a tumor suppressor in breast. Cell Death Differ 2008, 15:1752-1759.

10. Chan SW, Lim CJ, Guo K, Ng CP, Lee I, Hunziker W, Zeng Q, Hong W: A role for TAZ in migration, invasion, and tumorigenesis of breast cancer cells. Cancer Res 2008, 68:2592-2598.

11. Cordenonsi M, Zanconato F, Azzolin L, Forcato M, Rosato A, Frasson C, Inui M, Montagner M, Parenti AR, Poletti A, Daidone MG, Dupont S, Basso G, Bicciato S, Piccolo S: The Hippo transducer TAZ confers cancer stem cell-related traits on breast cancer cells. Cell 2011, 147:759-772.

12. Iorns E, Ward TM, Dean S, Jegg A, Thomas D, Murugaesu N, Sims D, Mitsopoulos C, Fenwick K, Kozarewa I, Naceur-Lombarelli C, Zvelebil M, Isacke CM, Lord CJ, Ashworth A, Hnatyszyn HJ, Pegram M, Lippman M: Whole genome in vivo RNAi screening identifies the leukemia inhibitory factor receptor as a novel breast tumor suppressor. Breast Cancer Res Treat 2012, 135:79-91

13. Chen D, Sun Y, Wei Y, Zhang P, Rezaeian AH, Teruya-Feldstein J, Gupta S, Liang $H$, Lin HK, Hung MC, Ma L: LIFR is a breast cancer metastasis suppressor upstream of the Hippo-YAP pathway and a prognostic marker. Nat Med 2012, 18:1511-1517.

14. Tamm C, Bower N, Anneren C: Regulation of mouse embryonic stem cell self-renewal by a Yes-YAP-TEAD2 signaling pathway downstream of LIF. J Cell Sci 2011, 124:1136-1144.

15. Lamar JM, Stern P, Liu H, Schindler JW, Jiang ZG, Hynes RO: The Hippo pathway target, YAP, promotes metastasis through its TEAD-interaction domain. Proc Natl Acad Sci U S A 2012, 109:E2441-2450.

16. Zhou D, Conrad C, Xia F, Park JS, Payer B, Yin Y, Lauwers GY, Thasler W, Lee JT, Avruch J, Bardeesy N: Mst1 and Mst2 maintain hepatocyte quiescence and suppress hepatocellular carcinoma development through inactivation of the Yap1 oncogene. Cancer Cell 2009, 16:425-438.

17. Matteucci E, Maroni P, Luzzati A, Perrucchini G, Bendinelli P, Desiderio MA: Bone metastatic process of breast cancer involves methylation state affecting E-cadherin expression through TAZ and WWOX nuclear effectors. Eur $J$ Cancer 2012 [Epub ahead of print].

18. Rowland BD, Peeper DS: KLF4, p21 and context-dependent opposing forces in cancer. Nat Rev Cancer 2006, 6:11-23.

19. Lai $D, H o K C$, Hao $Y$, Yang $X$ : Taxol resistance in breast cancer cells is mediated by the hippo pathway component TAZ and its downstream transcriptional targets Cyr61 and CTGF. Cancer Res 2011, 71:2728-2738.

doi:10.1186/bcr3349

Cite this article as: Hergovich A: YAP-Hippo signalling downstream of leukemia inhibitory factor receptor: implications for breast cancer. Breast Cancer Research 2012, 14:326. 